\title{
Insights into the paracrine effects of uterine natural killer cells
}

\author{
XIN GONG ${ }^{1}$, YANXIA LIU ${ }^{1}$, ZHENZHEN CHEN $^{2}$, CAI XU $^{1}$, QIUDAN LU $^{1}$ and ZHE JIN ${ }^{1}$ \\ ${ }^{1}$ Department of Reproductive Endocrinology, Dongfang Hospital of Beijing University of Chinese Medicine, Beijing 100078; \\ ${ }^{2}$ Department of Chinese Materia Medica, Beijing University of Chinese Medicine, Beijing 100102, P.R. China
}

Received January 22, 2014; Accepted August 11, 2014

DOI: $10.3892 / \mathrm{mmr} .2014 .2626$

\begin{abstract}
Uterine natural killer (uNK) cells are recruited into the uterus during establishment of the implantation and placentation of the embryo, and are hypothesized to regulate uterine spiral artery remodeling and angiogenesis during the initial stages of pregnancy. Failures in uNK cell activation are linked to diseases associated with pregnancy. However, the manner in which these cells interact with the endometrium remain unknown. Therefore, this study investigated the paracrine effects of uNK cells on the gene expression profile of an endometrial epithelial and stromal cell co-culture system in vitro, using a microarray analysis. Results from reverse transcription-quantitative polymerase chain reaction and enzyme-linked immunosorbent assay experiments showed that soluble factors from uNK cells significantly alter endometrial gene expression. In conclusion, this study suggests that paracrine effects of $\mathrm{uNK}$ cells guide $\mathrm{uNK}$ cell proliferation, trophoblast migration, endometrial decidualization and angiogenesis, and maintain non-cytotoxicity of uNK cells.
\end{abstract}

\section{Introduction}

In the human endometrium, uterine leukocytes undergo cyclic changes in cell number during the menstrual cycle. Uterine natural killer (uNK) cells comprise $70 \%$ of all decidual leukocytes during the secretory phase, when implantation occurs, and during early pregnancy $(1,2)$. At the initiation of embryo implantation and placentation, uNK cells interact with the extravillous trophoblasts and fetal cells that invade the uterus, where they remove and replace the smooth muscle of maternal spiral arteries (3). This ultimately converts small, coiled vessels into wider channels that are able to provide nutrients to the developing fetus (4).

Correspondence to: Professor Zhe Jin, Reproductive Endocrinology Centre, Dongfang Hospital of Beijing University of Chinese Medicine, 6 Fangxingyuan 1 Qu, Beijing 100078, P.R. China

E-mail: zjin0129@163.com

Key words: trophoblast invasion, spiral artery remodeling, decidua, human
The initial contact between the blastocyst and uterus occurs through adhesion of the embryonic trophectoderm to the uterine epithelium (5). During implantation, the epithelium is said to become 'receptive' (6). In addition, the transformation of endometrial stromal cells from small, densely packed cells to large polygonal cells with an open vesicular nucleus is one of the characteristic features of decidualization (7). These findings suggest that endometrial epithelial and stromal cells are important in implantation and decidualization. Furthermore, a previous study demonstrated that epithelial STAT3 controlled stromal function via a paracrine mechanism (8), indicating that there is epithelial-stromal crosstalk during implantation.

Implantation marks a transition stage in pregnancy, in which the blastocyst assumes a fixed position and establishes an altered physiological interaction with the uterus. The paracrine effects of uNK cells stimulate stromal fibroblasts to produce chemokines and cytokines, which support trophoblast migration during implantation. They also upregulate interleukin (IL)-15 and IL-15R $\alpha$ in stromal fibroblasts that may establish an environment for uNK cells to promote cell proliferation and recruitment into the uterus (9). There is strong evidence that implantation and early pregnancy are not a single event, and do not occur simultaneously (10). Thus, in order to investigate the effects of uNK cell paracrine signaling on these processes as a whole, a co-culture system consisting of epithelial and stromal cells was created. Furthermore, the regulation of trophoblast invasion and modification of the spiral arteries, was investigated (11).

\section{Materials and methods}

Ethical approval. All subjects understood and signed the informed consent form prior to participation. Experimental protocols were approved by the Ethics Committee of the Dongfang Hospital Human Ethics Committee, Beijing, China (no. 2011090201).

Tissue collection. Decidual tissues were obtained from ten healthy females undergoing an elective termination of a normal pregnancy at between seven and eight weeks of gestation, as determined by the last menstrual period.

Endometrial tissues were collected from biopsies taken during the proliferative phase of the menstrual cycle of females undergoing laparoscopy for benign disease (Dongfang Hospital of Beijing University of Chinese Medicine, Beijing, China). The 
exclusion criteria were hormonal stimulation, cancerous lesions and irregular menstrual bleeding. There were six volunteers and two endometrial biopsies per volunteer were obtained. Samples from three of the females (six biopsies) were used for the for microarray experiments and samples from the remaining three females (six biopsies) were used for the reverse transcription-quantitative polymerase chain reaction and enzyme-linked immunosorbent assay experiments. Of the two samples taken from each patient, one sample was used as a control and the other was used in the experimental group.

uNK cell isolation. uNK cells were purified as previously described (2). Briefly, decidual tissues were thoroughly washed with $\mathrm{Ca}^{2+}$ - and $\mathrm{Mg}^{2+}$-free Hank's balanced salt solution (HBSS) containing $100 \mathrm{U} / \mathrm{ml}$ penicillin and $100 \mathrm{~g} / \mathrm{ml}$ streptomycin (Sigma-Aldrich, St. Louis, MO, USA), cut into fragments of 1-2 $\mathrm{mm}^{3}$ using two scalpels and digested for $1 \mathrm{~h}$ at $37^{\circ} \mathrm{C}$ with gentle agitation in HBSS with $0.1 \%$ (w/v) collagenase I (Gibco-BRL, Carlsbad, CA, USA). Cell suspensions were layered over Ficoll-Hypaque medium (General Electric, Fairfield, CT, USA) and centrifuged at $800 \mathrm{x} \mathrm{g}$ for $25 \mathrm{~min}$. Cells at the interface were washed twice in RPMI-1640 media with $10 \%$ fetal calf serum (FCS) and antibiotics. Following incubation for $20 \mathrm{~min}$ at $4^{\circ} \mathrm{C}$ with anti-CD56 micro beads (Miltenyi Biotec GmbH, Bergisch Gladbach, Germany), cells were washed in washing buffer [phosphate-buffered saline (PBS), EDTA $2 \mathrm{mM}$ and $0.5 \%$ bovine serum albumin(w/v)] and loaded onto a manual cell separation (MS) column in a MiniMACS magnet (MiniMACS ${ }^{\mathrm{TM}}$ Separator System; Miltenyi Biotec $\mathrm{GmbH}$ ). The MS column was flushed three times and $\mathrm{CD}^{+} 6^{+}$cells were flushed according to the manufacturer's instructions. The purity of the uNK cells was $>90 \%$ $\mathrm{CD}^{\circ}{ }^{+} \mathrm{CD} 3^{-}$according to flow cytometric analysis. The uNK cells were cultured in RPMI-1640 media with 1\% FCS and 10 ng/ml IL-15 (R\&D Systems Inc., Minneapolis, MN, USA).

uNK cell-secretion medium production. uNK cell-secretion medium was prepared using $200 \mu \mathrm{l}$ RPMI-1640 media with $1 \%$ FCS and IL-15 (10 ng/ml) containing $5 \times 10^{5}$ of the purified uNK cells and placed into the upper chamber of a $0.4-\mu \mathrm{m}$ pore hanging cell culture insert (EMD Millipore, Billerica, MA, USA) in a 24-well tissue plate, with 1,300 $\mu \mathrm{l}$ of the same media excluding cells in the lower chamber. Germeyer et al (9) showed that soluble factors from uterine leucocytes had significant effects on endometrial cell gene expression. Thus, a hanging cell culture insert was used so that soluble molecules from the uNK cells were able pass through the filter into the lower chamber, without cells being in direct contact. The control medium comprised 1,500 $\mu \mathrm{l}$ RPMI-1640 media with $1 \%$ FCS and $10 \mathrm{ng} / \mathrm{ml}$ IL-15. This was used for subsequent experiments. Following incubation for $24 \mathrm{~h}$ at $37^{\circ} \mathrm{C}$, the uNK cell-secretion medium from the lower chamber and the control media were collected. To reduce interassay variability, the media from several batches was pooled for subsequent experiments and frozen at $-80^{\circ} \mathrm{C}$. Cells in the upper chamber were collected and the cell viability was measured using a live/dead viability kit (Invitrogen Life Technologies, Carlsbad, CA, USA). Only uNK cell samples containing $<35 \%$ of dead cells following overnight incubation were used for subsequent experiments.
Endometrial stromal and epithelial cell isolation. Human endometrial tissue was dissociated into single cells using $0.1 \%(\mathrm{w} / \mathrm{v})$ collagenase I (Life Technologies, Carlsbad, CA, USA) for 50-60 min at $37^{\circ} \mathrm{C}$. Cell suspensions were filtered using a $40-\mu \mathrm{m}$ sieve to separate undigested myometrial tissue and debris. Further dissociation of the filtrate was prevented by Dulbecco's modified Eagle's medium (DMEM)/F-12 (no Phenol Red; Gibco-BRL) with $10 \%$ FBS (Gibco-BRL). To remove erythrocytes, the cells were resuspended in $4 \mathrm{ml}$ DMEM/F12 with $1 \%$ FCS, layered over Ficoll-Paque PLUS (General Electric) and centrifuged for $25 \mathrm{~min}$ at $800 \mathrm{x} \mathrm{g}$. Endometrial cells were removed from the Ficoll-Paque PLUS medium interface, washed three times and resuspended in $1 \mathrm{ml}$ DMEM/F12 with $1 \%$ FCS. Leukocytes were removed with CD45-coated Dynabeads (Invitrogen Life Technologies). Purified stromal and epithelial cell suspensions were then obtained by a further round of magnetic bead sorting using Collection Epithelial Enrich Dynabeads (Invitrogen Life Technologies). Epithelial and stromal cell preparations were $>95 \%$ pure.

Stromal cells were cultured in DMEM/F12 with $10 \%$ FBS and an antibiotic-antimycotic agent $(100 \mathrm{U} / \mathrm{ml}$ penicillin, $100 \mathrm{~g} / \mathrm{ml}$ streptomycin, $10 \mu \mathrm{g} / \mathrm{ml}$ gentamicin $0.25 \mu \mathrm{g} / \mathrm{ml}$ amphotericin B; Life Technologies). Epithelial cells were cultured in serum-free bronchial epithelial cell growth medium (final volumes: $2 \mathrm{ml}$ bovine pituitary extract, $0.5 \mathrm{ml}$ insulin, $0.5 \mathrm{ml}$ $\mathrm{HC}, 0.5 \mathrm{ml}$ GA-1000, $0.5 \mathrm{ml}$ retinoic acid, $0.5 \mathrm{ml}$ transferrin, $0.5 \mathrm{ml}$ triiodothyronine, $0.5 \mathrm{ml}$ epinephrine and $0.5 \mathrm{ml}$ hEGF; Lonza, Walkersville, MD, USA) and an antibiotic-antimycotic agent. The isolated stromal and epithelial cells were separately seeded into six-well plates with $3 \mathrm{ml}$ culture medium per well. Each well contained stromal or epithelial cells from a single patient. After two weeks, cells were passaged into $25 \mathrm{~cm}^{2}$ cell culture flasks. Following this, stromal cells were passaged every 4-5 days and epithelial cells were passaged every 9-10 days.

Co-culture system. On day 20 following endometrial stromal and epithelial cell generation, cells of each type were seeded onto a Nunc UpCell Surface membrane (Thermo Labsystems, Santa Rosa, CA, USA). The co-culture system was built on these temperature-responsive cell culture surfaces, according to the manufacturer's instructions. In brief, when cells reached $80 \%$ confluence, all medium was aspirated and $500 \mu \mathrm{l}$ fresh medium was added. The membrane was then placed on top of the stromal cell layer. The Nunc UpCell Surface was maintained at $20^{\circ} \mathrm{C}$ for $13 \mathrm{~min}$. The membrane and cell layer were then carefully removed from the Nunc UpCell Surface using forceps. The membrane with the attached cell layer was transferred facing downwards onto the epithelial cell surface. Fresh medium was added and samples were incubated at $37^{\circ} \mathrm{C}$ for $40 \mathrm{~min}$. A further $1 \mathrm{ml}$ of medium was added to the top of the membrane and the membrane was withdrawn from the cell layer. The ratio of stromal to epithelial cells was 1:1, and every co-culture system was built using stromal and epithelial cells from the same participant. Co-cultured cells were maintained in DMEM/F12 with $1 \%$ FCS.

Co-culture system treatment. Each group, control and uNK cell, contained six co-culture systems. All the groups were washed twice with PBS and placed in serum-free DMEM for $16 \mathrm{~h}$ prior to subsequent experiments. DMEM was replaced 
Table I. Sequences of primers for reverse transcription-quantitative polymerase chain reaction.

\begin{tabular}{|c|c|c|c|}
\hline Gene & Primer sequence $5^{\prime} \rightarrow 3^{\prime}$ & Length (bases) & Amplicon (bp) \\
\hline \multirow[t]{2}{*}{ CXCL10 } & F: CTTTCTGACTCTAAGTGGCATTC & 23 & 176 \\
\hline & R: CACCCTTCTTTTTCATTGTAGCAA & 24 & \\
\hline \multirow[t]{2}{*}{ CXCL11 } & F: TATTACTATCTGTGGTTACGGTGGAG & 26 & 269 \\
\hline & R: GCACTTTTGCCAGTATCCCAT & 21 & \\
\hline \multirow[t]{2}{*}{ IL-15 } & F: TGGCTGCTGGAAACCC & 16 & 123 \\
\hline & R:CACAAGTAGCACTGGATGGAAAT & 23 & \\
\hline \multirow[t]{2}{*}{ SAMD9L } & F: GCCTTATCTCCACCTGTTTCTTAG & 24 & 300 \\
\hline & R: TGGGATGGCATTCCTTGAC & 19 & \\
\hline \multirow[t]{2}{*}{ GAPDH } & F: GAGCCAAAAGGGTCATCATCT & 21 & 231 \\
\hline & R: AGGGGCCATCCACAGTCTTC & 20 & \\
\hline
\end{tabular}

F, forward; R, reverse; CXCL, chemokine (C-X-C) motif ligand; IL-15, interleukin-15; SAMD9L, sterile $\alpha$ motif domain containing 9-like.

by $80 \%$ uNK cell-secretion medium and $20 \%$ DMEM in the uNK cell group, whilst the control group was treated with $80 \%$ control medium and 20\% DMEM. Following incubation for $6 \mathrm{~h}$, the cells and media from each group were collected. Three pairs of co-culture systems were used for the microarray studies and three pairs for the RT-qPCR experiments.

Microarray experiments. Total RNA was extracted from the endometrial cells in each co-culture system. RNA was purified with the RNeasy Mini kit (Qiagen, Hilden, Germany), according to the manufacturer's instructions. The microarray analysis was performed using the GeneChip ${ }^{\circledR}$ 3' IVT Express kit (Affymetrix Inc., Santa Clara, CA, USA). Briefly, total RNA underwent reverse transcription, first strand cDNA synthesis, double strand DNA, in vitro transcription, cRNA synthesis and fragmentation (12). Samples were hybridized onto GeneChip PrimeView Human Gene Expression Array (Affymetrix Inc.). This array covers $>36,000$ transcripts and variants. Following $16 \mathrm{~h}$ hybridization at $45^{\circ} \mathrm{C}$, arrays were washed on Fluidics Station 450 (Affymetrix, Inc.) and were scanned with Scanner 3000 (Affymetrix, Inc.) in order to obtain quantitative gene expression levels. The control and uNK cell groups were processed simultaneously throughout. Three chips were analyzed for each group.

$R T$-qPCR analysis. A total of four differentially expressed genes were selected for validation of the results from the microarray experiments using RT-qPCR. Cells from the control and $\mathrm{uNK}$ cell groups were washed twice with PBS, and total RNA was extracted using TRIzol (Life Technologies). Reverse transcription was performed with $8 \mu \mathrm{l}$ of total RNA per $20 \mu \mathrm{l}$ reaction using a standard cDNA Synthesis kit (Takara Bio, Inc., Otsu, Japan). The RT-qPCR primer sequences for target genes were self-designed by this group and ordered from Invitrogen. Primer sequences for target genes are shown in Table I.

For each RT-qPCR experiment, the typical thermal cycling conditions included an initial activation step at $95^{\circ} \mathrm{C}$ for $5 \mathrm{~min}$, 40 cycles at $95^{\circ} \mathrm{C}$ each for $30 \mathrm{sec}, 56^{\circ} \mathrm{C}$ for $20 \mathrm{sec}$ and $72^{\circ} \mathrm{C}$ for $30 \mathrm{sec}$. PCR reactions were performed on ABI Prism 7700 Sequence Detection system (Applied Biosystems Life
Technologies, Foster City, CA, USA). cDNA concentration was normalized to that of GAPDH. The target mRNA expression was analyzed using the $2^{-\Delta \Delta C t}$ algorithm.

ELISA experiments. IL-15 was analyzed using a commercially available ELISA kit (ELH-IL-15, RayBiotech, China) in the uNK cell-secretion medium prior to its use in the co-culture system experiments and in supernatants of the co-culture systems that had been treated with control media or with uNK cell-secretion medium. The analysis was conducted according to the manufacturer's instructions. Assays were performed in triplicate and concentrations of IL-15 ( $\mathrm{pg} / \mathrm{ml})$ were compared with standard curves. To determine the quantities of IL-15 secreted by the co-culture systems, the starting IL-15 content of the uNK cell-secretion medium was subtracted. The sensitivity of the kit was $10 \mathrm{pg} / \mathrm{ml}$.

Statistical analysis. Data were analyzed using analysis of variance using SPSS 17.0 software (SPSS, Inc., Chicago, IL, USA). In the microarray experiments, median fold change ratios between the control and uNK cell groups were derived for each transcript, and genes that were up- or downregulated with a fold change $>1$ and $\mathrm{P}<0.001$ were selected. In RT-qPCR and ELISA analysis, data are presented as the median \pm standard error of the mean. $\mathrm{P}<0.01$ was considered to indicate a statistically significant difference. Graphs of the data were produced using Microsoft Excel software.

\section{Results}

Microarray experiments. Gene expression profiling using a microarray was used to compare transcript expression in the endometrial co-culture systems treated with either control medium or uNK cell-secretion medium.

This analysis identified 155 upregulated genes that exhibited a change of $>2$-fold in the median expression level in response to uNK cell-secretion medium. No transcript was downregulated $>2$-fold (Table II). However, certain genes in uNK cell groups were upregulated with a 1.0-2.0-fold change, compared with the control group. Previous studies have shown 
Table II. Upregulated transcripts altered $>2$-fold.

\begin{tabular}{llll}
\hline Gene & Fold change & Gene ID & Description
\end{tabular}

Cytokines/ Chemokines

CXCL10

11.1

CXCL11

IL-15

IL-7

Immunological factors

$$
\text { NLRC5 }
$$

FAM111A

ACTR2

HSPH1

IFIT5

UVRAG

Apoptotic protein

RASSF6

Tryptophan metabolism

IDO1

Signaling factors

$$
\text { GBP2 }
$$

UACA

\section{IFIT3 \\ ALCAM}

CGA

EDNRA

WASF2

IL6ST

USP15

MIER1

RGS12

Transcription

STAT1
IRF1
IRF9
IFI16
ATRX
TCERG1
ZNF644
ZEB1
SAFB2
PRDM2

Nucleotide metabolism

$$
\text { NUFIP2 }
$$

PAPOLA

EIF4G1

CRCP

AGGF1

XRN2

Enzyme activity

GBP4

GBP5
Chemokine (C-X-C motif) ligand 10 Chemokine (C-X-C motif) ligand 11 Interleukin 15 Interleukin 7

NLR family, CARD domain containing 5

Family with sequence similarity 111 , member A

ARP2 actin-related protein 2 homolog (yeast)

Heat shock protein 1

Interferon-induced protein with tetratricopeptide repeats 5 $\mathrm{UV}$ radiation resistance associated gene

Ras association (RalGDS/AF-6) domain family member 6

Indoleamine 2,3-dioxygenase 1

Guanylate binding protein 2, interferon-inducible

Uveal autoantigen with coiled-coil domains and ankyrin repeats

Interferon-induced protein with tetratricopeptide repeats 3 Activated leukocyte cell adhesion molecule

Glycoprotein hormones, $\alpha$ polypeptide

Endothelin receptor type A

WAS protein family, member 2

Interleukin 6 signal transducer

Ubiquitin specific peptidase 15

Mesoderm induction early response 1 homolog

Regulator of G-protein signaling 12

Signal transducer and activator of transcription 1 Interferon regulatory factor 1

Interferon regulatory factor 9

Interferon, $\gamma$-inducible protein 16

Alpha thalassemia/mental retardation syndrome X-linked

Transcription elongation regulator 1

Zinc finger protein 644

Zinc finger E-box binding homeobox 1

Scaffold attachment factor B2

PR domain containing 2

Nuclear fragile $\mathrm{X}$ mental retardation protein interacting protein 2

Poly(A) polymerase alpha

Eukaryotic translation initiation factor $4 \gamma, 1$

CGRP receptor component

Angiogenic factor with $G$ patch and FHA domains 1

5'-3' exoribonuclease 2

Guanylate binding protein 4

Guanylate binding protein 5 
Table II. Continued.

\begin{tabular}{|c|c|c|c|}
\hline Gene & Fold change & Gene ID & Description \\
\hline INPP4B & 4.8 & 8821 & Inositol polyphosphate-4-phosphatase, type II \\
\hline GBP7 & 4.7 & 388646 & Guanylate binding protein 7 \\
\hline SETD2 & 4.2 & 29072 & SET domain containing 2 \\
\hline GBP1 & 3.9 & 2633 & Guanylate binding protein 1 \\
\hline PSMB9 & 3.3 & 5698 & Proteasome (prosome, macropain) subunit, $\beta$ type, 9 \\
\hline WASL & 3.3 & 8976 & Wiskott-Aldrich syndrome-like \\
\hline PUS7L & 3.0 & 83448 & Pseudouridylate synthase 7 homolog-like \\
\hline C1R & 2.9 & 715 & Complement component 1 \\
\hline TAP1 & 2.8 & 6890 & Transporter 1, ATP-binding cassette, sub-family B (MDR/TAP) \\
\hline FAF2 & 2.7 & 23197 & Fas associated factor family member 2 \\
\hline UBE2L6 & 2.7 & 9246 & Ubiquitin-conjugating enzyme E2L 6 \\
\hline PARP14 & 2.7 & 54625 & Poly (ADP-ribose) polymerase family, member 14 \\
\hline PARP9 & 2.7 & 83666 & Poly (ADP-ribose) polymerase family, member 9 \\
\hline PSME4 & 2.5 & 23198 & Proteasome (prosome, macropain) activator subunit 4 \\
\hline OTUD4 & 2.5 & 54726 & OTU domain containing 4 \\
\hline BIRC6 & 2.4 & 57448 & Baculoviral IAP repeat containing 6 \\
\hline ARHGAP21 & 2.4 & 57584 & Rho GTPase activating protein 21 \\
\hline DTX3L & 2.4 & 151636 & Deltex 3-like \\
\hline RARRES3 & 2.3 & 5920 & Retinoic acid receptor responder 3 \\
\hline MGEA5 & 2.3 & 10724 & Meningioma expressed antigen 5 \\
\hline DCAF8 & 2.3 & 50717 & DDB1 and CUL4 associated factor 8 \\
\hline GPD2 & 2.3 & 2820 & Glycerol-3-phosphate dehydrogenase 2 \\
\hline UHMK1 & 2.3 & 127933 & U2AF homology motif (UHM) kinase 1 \\
\hline PDP1 & 2.2 & 54704 & Pyruvate dehyrogenase phosphatase catalytic subunit 1 \\
\hline USP10 & 2.2 & 9100 & Ubiquitin specific peptidase 10 \\
\hline COIL & 2.2 & 8161 & Coilin \\
\hline UFL1 & 2.2 & 23376 & UFM1-specific ligase 1 \\
\hline USP1 & 2.1 & 7398 & Ubiquitin specific peptidase 1 \\
\hline G2E3 & 2.1 & 55632 & G2/M-phase specific E3 ubiquitin protein ligase \\
\hline NF1 & 2.0 & 4763 & Neurofibromin 1 \\
\hline PHACTR2 & 2.0 & 9749 & Phosphatase and actin regulator 2 \\
\hline \multicolumn{4}{|l|}{ Transporters } \\
\hline TPR & 4.4 & 7175 & Translocated promoter region, nuclear basket protein \\
\hline APOL6 & 3.2 & 80830 & Apolipoprotein L, 6 \\
\hline GCC2 & 2.8 & 9648 & GRIP and coiled-coil domain containing 2 \\
\hline APOL3 & 2.4 & 80833 & Apolipoprotein L, 3 \\
\hline CPNE3 & 2.4 & 8895 & Copine III \\
\hline USO1 & 2.2 & 8615 & USO1 vesicle docking protein homolog \\
\hline SLC38A1 & 2.2 & 81539 & Solute carrier family 38, member 1 \\
\hline NIPAL1 & 2.1 & 152519 & NIPA-like domain containing 1 \\
\hline KIAA1033 & 2.1 & 23325 & KIAA1033 \\
\hline NUPL1 & 2.1 & 9818 & Nucleoporin like 1 \\
\hline \multicolumn{4}{|c|}{ Structural factors } \\
\hline MIS18BP1 & 3.9 & 55320 & MIS18 binding protein 1 \\
\hline CDC27 & 3.3 & 996 & Cell division cycle 27 homolog \\
\hline CALD1 & 3.1 & 800 & Caldesmon 1 \\
\hline LIMA1 & 3.0 & 51474 & LIM domain and actin binding 1 \\
\hline SLMAP & 2.7 & 7871 & Sarcolemma associated protein \\
\hline TLN1 & 2.7 & 7094 & Talin 1 \\
\hline EZR & 2.5 & 7430 & Ezrin \\
\hline ITGB 1 & 2.5 & 3688 & Integrin, $\beta 1$ \\
\hline
\end{tabular}


Table II. Continued.

\begin{tabular}{|c|c|c|c|}
\hline Gene & Fold change & Gene ID & Description \\
\hline APPL1 & 2.5 & 26060 & $\begin{array}{l}\text { Adaptor protein, phosphotyrosine interaction, } \\
\text { PH domain and leucine zipper containing } 1\end{array}$ \\
\hline RTP4 & 2.5 & 64108 & Receptor (chemosensory) transporter protein 4 \\
\hline WAPAL & 2.4 & 23063 & Wings apart-like homolog \\
\hline PRRC2C & 2.4 & 23215 & Proline-rich coiled-coil 2C \\
\hline CASC5 & 2.4 & 57082 & Cancer susceptibility candidate 5 \\
\hline ENC1 & 2.3 & 8507 & Ectodermal-neural cortex 1 \\
\hline KIF14 & 2.2 & 9928 & Kinesin family member 14 \\
\hline SPTBN1 & 2.2 & 6711 & Spectrin, $\beta$, non-erythrocytic 1 \\
\hline MYO6 & 2.2 & 4646 & Myosin VI \\
\hline ODF2L & 2.1 & 57489 & Outer dense fiber of sperm tails 2-like \\
\hline DYNC1H1 & 2.1 & 1778 & Dynein, cytoplasmic 1 , heavy chain 1 \\
\hline PPP4R2 & 2.1 & 151987 & Protein phosphatase 4 , regulatory subunit 2 \\
\hline SRPR & 2.0 & 6734 & Signal recognition particle receptor \\
\hline \multicolumn{4}{|l|}{ Kinase } \\
\hline WNK1 & 4.0 & 65125 & WNK lysine deficient protein kinase 1 \\
\hline PRKDC & 3.5 & 5591 & Protein kinase, DNA-activated, catalytic polypeptide \\
\hline IQGAP1 & 3.0 & 8826 & IQ motif containing GTPase activating protein 1 \\
\hline CMPK2 & 2.8 & 129607 & $\begin{array}{l}\text { Cytidine monophosphate (UMP-CMP) kinase } 2 \text {, } \\
\text { mitochondrial }\end{array}$ \\
\hline BAZ1B & 2.7 & 9031 & Bromodomain adjacent to zinc finger domain, 1B \\
\hline CCND2 & 2.6 & 894 & Cyclin D2 \\
\hline MOB1A & 2.5 & 55233 & MOB kinase activator $1 \mathrm{~A}$ \\
\hline MAP4K5 & 2.5 & 11183 & $\begin{array}{l}\text { Mitogen-activated protein kinase kinase kinase } \\
\text { kinase } 5\end{array}$ \\
\hline \multicolumn{4}{|c|}{ Ion binding proteins } \\
\hline DSC2 & 4.5 & 1824 & Desmocollin 2 \\
\hline EEA1 & 4.2 & 8411 & Early endosome antigen 1 \\
\hline ZC3H11A & 3.3 & 9877 & Zinc finger CCCH-type containing 11A \\
\hline CLSTN1 & 2.8 & 22883 & Calsyntenin 1 \\
\hline $\mathrm{C} 1 \mathrm{~S}$ & 2.7 & 716 & Complement component 1 , s subcomponent \\
\hline THAP6 & 2.6 & 152815 & THAP domain containing 6 \\
\hline RSAD2 & 2.5 & 91543 & Radical S-adenosyl methionine domain containing 2 \\
\hline ZNFX1 & 2.3 & 57169 & Zinc finger, NFX1-type containing 1 \\
\hline PLCB4 & 2.2 & 5332 & Phospholipase C, $\beta 4$ \\
\hline TIPARP & 2.2 & 25976 & TCDD-inducible poly(ADP-ribose) polymerase \\
\hline WDFY1 & 2.1 & 57590 & WD repeat and FYVE domain containing 1 \\
\hline CHURC1 & 2.1 & 91612 & Churchill domain containing 1 \\
\hline ITGA4 & 2.1 & 3676 & Integrin, $\alpha 4$ \\
\hline XAF1 & 2.1 & 54739 & XIAP associated factor 1 \\
\hline \multicolumn{4}{|c|}{ DNA/RNA proteins } \\
\hline ZFR & 4.4 & 51663 & Zinc finger RNA binding protein \\
\hline TOP1 & 4.2 & 7150 & Topoisomerase (DNA) I \\
\hline BOD1L1 & 3.4 & 259282 & Biorientation of chromosomes in cell division 1-like 1 \\
\hline IFIT2 & 3.3 & 3433 & Interferon-induced protein with tetratricopeptide repeats 2 \\
\hline CENPF & 3.1 & 1063 & Centromere protein F, 350/400 kDa (mitosin) \\
\hline MBNL1 & 3.0 & 4154 & Muscleblind-like splicing regulator 1 \\
\hline DHX9 & 2.4 & 1660 & DEAH (Asp-Glu-Ala-His) box polypeptide 9 \\
\hline FMR1 & 2.4 & 2332 & Fragile X mental retardation 1 \\
\hline DDX58 & 2.3 & 23586 & DEAD (Asp-Glu-Ala-Asp) box polypeptide 58 \\
\hline BCLAF1 & 2.3 & 9774 & BCL2-associated transcription factor 1 \\
\hline DNMT1 & 2.2 & 1786 & DNA (cytosine-5-)-methyltransferase 1 \\
\hline
\end{tabular}


Table II. Continued.

\begin{tabular}{|c|c|c|c|}
\hline Gene & Fold change & Gene ID & Description \\
\hline NOL8 & 2.2 & 55035 & Nucleolar protein 8 \\
\hline RNF213 & 2.2 & 57674 & Ring finger protein 213 \\
\hline BDP1 & 2.2 & 55814 & B double prime 1 \\
\hline RAD21 & 2.2 & 5885 & RAD21 homolog \\
\hline HP1BP3 & 2.1 & 50809 & Heterochromatin protein 1 , binding protein 3 \\
\hline SF3B 1 & 2.0 & 23451 & Splicing factor $3 \mathrm{~b}$, subunit 1 \\
\hline \multicolumn{4}{|l|}{ Other } \\
\hline SAMD9L & 5.7 & 219285 & Sterile $\alpha$ motif domain containing 9-like \\
\hline C10orf118 & 5.5 & 55088 & Chromosome 10 open reading frame 118 \\
\hline MTUS1 & 2.7 & 57509 & Microtubule associated tumor suppressor 1 \\
\hline EPSTI1 & 2.6 & 94240 & Epithelial stromal interaction 1 \\
\hline ATXN7L3B & 2.6 & 552889 & Ataxin 7-like 3B \\
\hline ANKRD32 & 2.5 & 84250 & Ankyrin repeat domain 32 \\
\hline EHBP1 & 2.4 & 23301 & EH domain binding protein 1 \\
\hline PPFIBP1 & 2.4 & 8496 & PTPRF interacting protein, binding protein 1 (liprin $\beta 1$ ) \\
\hline TMTC3 & 2.3 & 160418 & Transmembrane and tetratricopeptide repeat containing 3 \\
\hline CEP350 & 2.2 & 9857 & Centrosomal protein $350 \mathrm{kDa}$ \\
\hline BTBD10 & 2.2 & 84280 & BTB (POZ) domain containing 10 \\
\hline BTN3A3 & 2.1 & 10384 & Butyrophilin, subfamily 3, member A3 \\
\hline KCTD9 & 2.1 & 54793 & Potassium channel tetramerisation domain containing 9 \\
\hline
\end{tabular}

Gene transcription in co-culture systems following stimulation with uNK cell-secretion medium using a GeneChip PrimeView Human Gene Expression Array. $\mathrm{P}<0.001$, compared with control group. Upregulated genes: 155 transcripts. uNK, uterine natural killer cells.
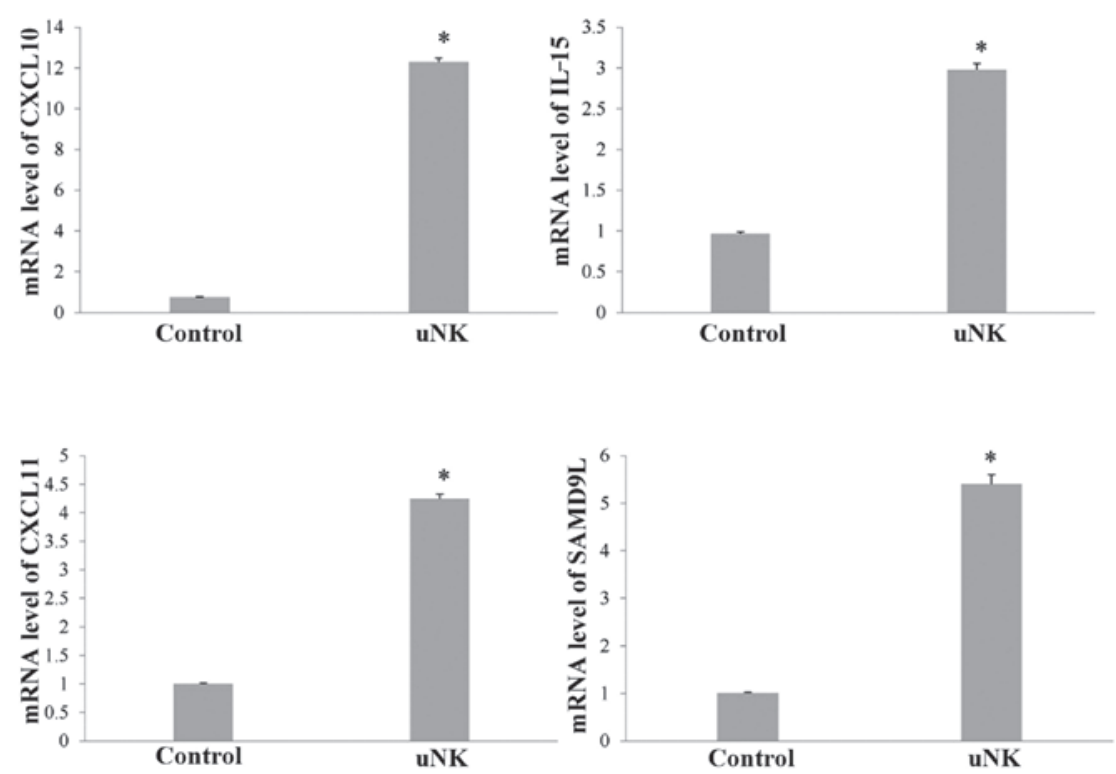

Figure 1. Transcript levels of CXCL10, CXCL11, IL-15 and SAMD9L determined by RT-qPCR. The RT-qPCR results were consistent with those of the microarray analysis. Data are expressed as the mean \pm standard error of the mean. " $\mathrm{P}<0.01$, compared with the control group. uNK, uterine natural killer cells; CXCL, chemokine (C-X-C) motif ligand; IL-15, interleukin 15; SAMD9L, sterile $\alpha$ motif domain containing 9-like; RT-qPCR, reverse transcription-quantitative polymerase chain reaction.

that these genes have an important functions in uNK cells. For instance, the co-culture system treated with uNK cell-secretion medium showed increased expression of interleukin (IL)15RA (1.6-fold) (13), vascular endothelial growth factor (VEGF)-C (1.35-fold) (14), intercellular adhesion molucule (ICAM) 1 (1.66-fold) (15), superoxide dismutase (SOD)2 (1.09-fold), caspase (CASP)1 (1.85-fold), nuclear factor erythroid 2-related factor 3 (NFE2L3) (1.37-fold), interferon $\gamma$ receptor 1 (IFNGR1; 


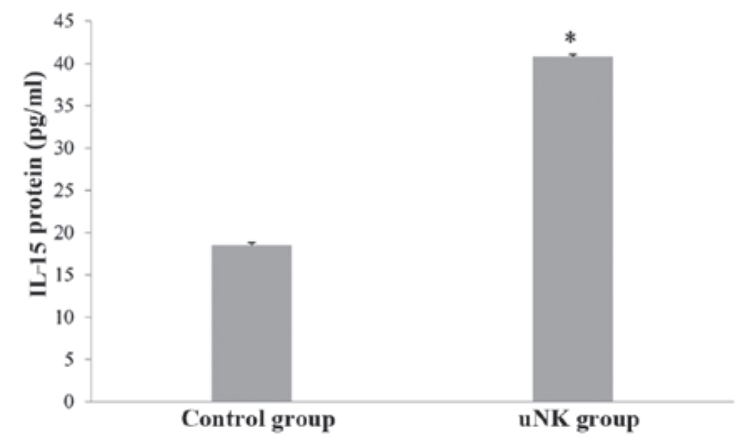

Figure 2. IL-15 levels in control and uNK groups. An enzyme-linked immunosorbent assay was used to measure IL-15 levels in supernatants from co-culture systems treated for $6 \mathrm{~h}$ with conditioned medium from decidual uNK cells or with control medium. Data are presented as the mean \pm standard error of the mean. Values were corrected for the quantity of IL-15 detected in the control and uNK cell-secretion medium prior to addition to the co-culture systems. ${ }^{*} \mathrm{P}<0.01$, compared with the control group. IL-15, interleukin 15 ; uNK, uterine natural killer cells

1.10-fold) (16), major histocompatibility complex (MHC) class I polypeptide-related sequence A (1.12-fold) and MHC class I polypeptide-related sequence B $(1.34$-fold) $(3,17)$ and showed decreased expression of IGFBP3 (1.04-fold) $(\mathrm{P}<0.001)$.

$R T$-qPCR analysis. In order to verify these changes, transcript levels for certain genes were measured by RT-qPCR, including chemokine (C-X-C) motif ligand (CXCL)10, CXCL11, IL-15 and sterile $\alpha$ motif domain containing 9-like (SAMD9L; Fig. 1). The RT-qPCR results confirmed the significant changes in expression that had been indicated by the microarray analysis. The endometrial co-culture system treated with uNK cell-secretion medium showed increased expression of CXCL10 (16.4-fold), CXCL11 (4.3-fold), IL-15 (3.1-fold) and SAMD9L (5.4-fold; $\mathrm{P}<0.01)$.

ELISA experiments. To confirm the observed changes in the IL-15 protein level, the quantity of IL-15 was analyzed by ELISA in the supernatant of the control and uNK cell-secretion medium-stimulated endometrial co-culture systems. There was a significant increase in IL-15 protein levels in the experimental groups compared with the control group (Fig. 2; $\mathrm{P}<0.01)$.

\section{Discussion}

Several observations suggest that uNK cells are involved in reproduction. They increase in number during the luteal period of the menstrual cycle when implantation occurs (1). They are present in the early phases of gestation, when placental cells invade into the maternal arteries (18). In addition, they are particularly abundant in the area surrounding the infiltrating fetally derived extravillous cells (19). During the progesterone-dominated phase of the menstrual cycle, uNK cells show changes in the levels of transcripts for VEGF-C (20). Previous protein array studies have shown that uNK cells are the predominant producers of angiogenic growth factors in early pregnancy (21). In addition, in an ex vivo chorionic plate artery model, uNK cells promoted vessel-like assembly of extravillous cytotrophoblast cell lines $(15,22)$. Insufficient
$\mathrm{uNK}$ cell activation may reduce these processes and contribute to poor arterial remodeling in decidua, thus increasing the risk of preeclampsia and intrauterine fetal growth restriction (23).

In humans, CD56 ${ }^{+} \mathrm{NK}$ cells are associated with the synthesis of immunoregulatory cytokines, particularly IFN- $\gamma$ (24). IFN- $\gamma$ significantly upregulates certain chemokines [CXCL9, CXCL10, chemokine (C-C motif) ligand 8 and IL-15R $\alpha$ ], enzymes [guanylate binding protein 5, transporter associated with antigen processing (TAP1), SOD2 and CASP1] and transcription factors (interferon regulatory factor 1, NFE2L3 and transcription factor AP-2 $\gamma$ ). It is also known to downregulate insulin-like growth factor binding proteins (Wnt1 inducible signaling pathway 2 and insulin-like growth factor-binding protein 3) (16). These actions, combined with the $\mathrm{uNK}$ cell production of chemokines CXCL10 and CXCR2, direct the migration and invasion of trophoblasts (25) and promote angiogenesis in the placental bed $(26,27)$. The present study found similar changes in gene expression, for example, IFNGR1 transcript levels were significantly increased in co-culture systems stimulated by uNK cell-secretion medium. This concordance strongly suggests that uNK cell paracrine signaling, combined with INF- $\gamma$, regulates the expression of genes involved in embryo and trophoblast migration, endometrial decidualization and angiogenesis in human uterine endometrium.

Implantation-associated decidualization in the rat and mouse results in the accumulation of NK cells in the uterine mesometrial decidua (28). uNK cells are hypothesized to be involved in pregnancy-associated uterine vascular development (20). However, it is not clear how uNK cells communicate with the developing endometrial cells in order to facilitate this process. Previous in vitro experimentation has indicated that uNK cells produce factors that directly affect the behavior of trophoblast cells (25). A number of studies have suggested that uNK cell supernatant stimulate trophoblast invasion (29), whereas others have concluded that it is the uNK cell supernatants that stimulate these process (20). The results of the current study indicate that the paracrine effects of uNK cells on endometrial epithelial and stromal cells mediate the development of the vasculature. Levels of ICAM-1, which is involved the migration and network formation of the trophoblast cell line (15), increased significantly in this study. Thus, it is likely that ICAM-1 is involved in this paracrine network.

Although replete with cytotoxic machinery, uNK cells remain tolerant at the maternal-fetal interface (26). A previous indicated that this is facilitated by VEGF-C (30), and in vitro studies have also suggested that the involvement of IL-15 is important in promoting this tolerance (17). In addition, the non-cytotoxic capacity of uNK cells is based on their ability to recognize surface MHC class I molecules on target cells, which deliver signals that suppress NK cell function. A number of studies have suggested that a lack of engagement of MHC-specific receptors leads to NK cell-mediated killing $(17,31)$. The results from this study showed that there was an increased expression of VEGF-C, IL-15 and MHC class I polypeptide-related sequence $\mathrm{A} / \mathrm{B}$ in the co-culture systems that were treated with uNK cell-secretion medium compared with the control group, indicating similar non-cytotoxic mechanism to those already postulated. Furthermore, there is evidence that endothelial cells exhibit sensitivity to activated peripheral blood NK cells in the 
absence of the expression of TAP1 (32), as TAP-1 is a key factor essential for peptide loading for MHC class I assembly (33). It is proposed that VEGF-C is the predominant regulator of TAP1 expression in the uterus (30), and the present study showed higher TAP1 expression in the uNK cell group. These findings support a dual function of VEGF-C, in which it acts as an angiogenic factor and also promotes immune tolerance in the uterine microenvironment. To the best of our knowledge, this is the first evidence that noncytotoxicity of uNK cells is directly coupled to their vascular remodeling and angiogenesis functions.

The origins of human uNK cells are not clear, and a number of mechanisms have been postulated (34-36). Several of the molecules that were altered by uNK cell-secretion medium in this study are known to have important functions in $\mathrm{NK}$ cell proliferation, including IL-15 and IL-15R $\alpha$. IL-15 has a variety of functions, including the induction of $\mathrm{T}$ cell proliferation and the activation of cytotoxic effector cells and monocytes $(37,38)$. The reciprocal interactions between uNK cells and the epithelial/stromal cell co-culture system observed in this study are similar to those in bone marrow during NK cell development. NK cells upregulate IL-15 in the bone marrow microenvironment, which is then bound and presented by IL-15R $\alpha$ to the stromal cell surface, promoting increased NK cell proliferation (39). These results suggest that this mechanism may occur in the endometrium in response to molecules secreted by uNK cells. The results suggest that $\mathrm{uNK}$ cells and non-decidualized stromal and epithelial cells may interact to maintain immune cell homeostasis in the endometrial microenvironment.

In conclusion, to the best of our knowledge, this is the first detailed study of the paracrine interaction between uNK cells and endometrial cells (epithelial/stromal cells). It indicates that this paracrine signaling may contribute to $\mathrm{UNK}$ cell proliferation and recruitment, embryo and trophoblast migration, endometrial decidualization, angiogenesis and immune tolerance in the uterine microenvironment.

\section{Acknowledgements}

This study was supported by the National Natural Science Foundation of China (grant no. 81173292).

\section{References}

1. King A, Wellings V, GardnerLandLoke YW:Immunocytochemica characterization of the unusual large granular lymphocytes in human endometrium throughout the menstrual cycle. Hum Immunol 24: 195-205, 1989.

2. Verma S, Hiby SE, Loke YW and King A: Human decidual natural killer cells express the receptor for and respond to the cytokine interleukin 15. Biol Reprod 62: 959-968, 2000.

3. Moffett A and Loke C: Immunology of placentation in eutherian mammals. Nat Rev Immunol 6: 584-594, 2006.

4. Parham P and Guethlein LA: Pregnancy immunogenetics: NK cell education in the womb? J Clin Invest 120: 3801-3804, 2010.

5. Carson DD, Wilson OF and Dutt A: Glycoconjugate expression and interactions at the cell surface of mouse uterineu epithelial cells and periimplantation-stage embryos. In: Trophoblast Invasion and Endometrial Receptivity. Springer, New York, NY, USA, pp211-241, 1990.

6. Murphy CR and Shaw TJ: Plasma membrane transformation: a common response of uterine epithelial cells during the peri-implantation period. Cell Biol Int 18: 1115-1128, 1994.

7. Inoue $\mathrm{T}$, Kanzaki H, Iwai M, et al: Tumour necrosis factor alpha inhibits in-vitro decidualization of human endometrial stromal cells. Hum Reprod 9: 2411-2417, 1994.
8. Pawar S, Starosvetsky E, Orvis GD, Behringer RR, Bagchi IC and Bagchi MK: STAT3 regulates uterine epithelial remodeling and epithelial-stromal crosstalk during implantation. Mol Endocrinol 27: 1996-2012, 2013.

9. Germeyer A, Sharkey AM, Prasadajudio M, et al: Paracrine effects of uterine leucocytes on gene expression of human uterine stromal fibroblasts. Mol Hum Reprod 15: 39-48, 2009.

10. Schlafke S and Enders AC: Cellular basis of interaction between trophoblast and uterus at implantation. Biol Reprod 12: 41-65, 1975.

11. Hiby SE, Walker JJ, O'shaughnessy KM, et al: Combinations of maternal KIR and fetal HLA-C genes influence the risk of preeclampsia and reproductive success. J Exp Med 200: 957-965, 2004.

12. Gong X, Chen Z, Liu Y, Lu Q and Jin Z: Gene expression profiling of the paracrine effects of uterine natural killer cells on human endometrial epithelial cells. Int J Endocrinol 2014: 393707, 2014.

13. Grabstein KH, Eisenman J, Shanebeck K, et al: Cloning of a $\mathrm{T}$ cell growth factor that interacts with the beta chain of the interleukin-2 receptor. Science 264: 965-968, 1994.

14. Zhou Y, Bellingard V, Feng KT, McMaster M and Fisher SJ: Human cytotrophoblasts promote endothelial survival and vascular remodeling through secretion of Ang2, PlGF, and VEGF-C. Dev Biol 263: 114-125, 2003.

15. Hu Y, Eastabrook G, Tan R, MacCalman C, Dutz JP and von Dadelszen P: Decidual NK cell-derived conditioned medium enhances capillary tube and network organization in an extravillous cytotrophoblast cell line. Placenta 31: 213-221, 2010.

16. Kitaya K, Yasuo T, Yamaguchi T, Fushiki S and Honjo H: Genes regulated by interferon- $\gamma$ in human uterine microvascular endothelial cells. Int J Mol Med 20: 689-697, 2007.

17. Cooper MA, Fehniger TA and Caligiuri MA: The biology of human natural killer-cell subsets. Trends Immunol 22: 633-640, 2001.

18. Bulmer J, Johnson P and Bulmer D: Leukocyte populations in human decidua and endometrium. In: Immunoregulation and Fetal Survival. Oxford University Press, Oxford, UK, pp111-134, 1987.

19. Loke Y: Human implantation: Cell Biology and Immunology. Cambridge University Press, Cambridge, UK, 1995.

20. Zhang J, Chen Z, Smith GN and Croy BA: Natural killer cell-triggered vascular transformation: maternal care before birth? Cell Mol Immunol 8: 1-11, 2011

21. Lash GE, Robson SC and Bulmer JN: Review: Functional role of uterine natural killer ( $\mathrm{uNK}$ ) cells in human early pregnancy decidua. Placenta 31 (Suppl): S87-S92, 2010.

22. Hu Y, Dutz JP, MacCalman CD, Yong $\mathrm{P}$, Tan $\mathrm{R}$ and von Dadelszen P: Decidual NK cells alter in vitro first trimester extravillous cytotrophoblast migration: a role for IFN-gamma. J Immunol 177: 8522-8530, 2006.

23. Wegmann TG, Lin H, Guilbert L and Mosmann TR: Bidirectional cytokine interactions in the maternal-fetal relationship: is successful pregnancy a TH2 phenomenon? Immunol Today 14: 353-356, 1993.

24. Ottaviani C, Nasorri F, Bedini C, de Pità O, Girolomoni G and Cavani A: CD56brightCD16(-) NK cells accumulate in psoriatic skin in response to CXCL10 and CCL5 and exacerbate skin inflammation. Eur J Immunol 36: 118-128, 2006.

25. Hanna J, Goldman-Wohl D, Hamani Y, et al: Decidual NK cells regulate key developmental processes at the human fetal-maternal interface. Nat Med 12: 1065-1074, 2006.

26. Moffett-King A: Natural killer cells and pregnancy. Nat Rev Immunol 2: 656-663, 2002.

27. Wang A, Rana S and Karumanchi SA: Preeclampsia: the role of angiogenic factors in its pathogenesis. Physiology (Bethesda) 24: 147-158, 2009.

28. Ain R, Canham LN and Soares MJ: Gestation stage-dependent intrauterine trophoblast cell invasion in the rat and mouse: novel endocrine phenotype and regulation. Dev Biol 260: 176-190, 2003.

29. Smith SD, Dunk CE, Aplin JD, Harris LK and Jones RL: Evidence for immune cell involvement in decidual spiral arteriole remodeling in early human pregnancy. Am J Pathol 174: 1959-1971, 2009

30. Kalkunte SS, Mselle TF, Norris WE, Wira CR, Sentman CL and Sharma S: Vascular endothelial growth factor C facilitates immune tolerance and endovascular activity of human uterine NK cells at the maternal-fetal interface. J Immunol 182: 4085-4092, 2009.

31. Riley JK and Yokoyama WM: NK cell tolerance and the maternal-fetal interface. Am J Reprod Immunol 59: 371-387, 2008. 
32. Ayalon O, Hughes EA, Cresswell P, et al: Induction of transporter associated with antigen processing by interferon gamma confers endothelial cell cytoprotection against natural killer-mediated lysis. Proc Natl Acad Sci USA 95: 2435-2440, 1998.

33. Cox JH, Yewdell JW, Eisenlohr LC, Johnson PR and Bennink JR: Antigen presentation requires transport of MHC class I molecules from the endoplasmic reticulum. Science 247: 715-718, 1990.

34. Vosshenrich CA, García-Ojeda ME, Samson-Villéger SI, et al: A thymic pathway of mouse natural killer cell development characterized by expression of GATA-3 and CD127. Nat Immunol 7: 1217-1224, 2006

35. van den Heuvel M, Peralta C, Bashar S, Taylor S, Horrocks J and Croy BA: Trafficking of peripheral blood CD56(bright) cells to the decidualizing uterus - new tricks for old dogmas? J Reprod Immunol 67: 21-34, 2005.
36. Gargett CE, Schwab KE, Zillwood RM, Nguyen HP and Wu D: Isolation and culture of epithelial progenitors and mesenchymal stem cells from human endometrium. Biol Reprod 80: 1136-1145, 2009.

37. Carson WE, Giri JG, Lindemann MJ, et al: Interleukin (IL) 15 is a novel cytokine that activates human natural killer cells via components of the IL-2 receptor. J Exp Med 180: 1395-1403, 1994.

38. Waldmann $\mathrm{T}$ and Tagaya $\mathrm{Y}$ : The multifaceted regulation of interleukin-15 expression and the role of this cytokine in NK cell differentiation and host response to intracellular pathogens. Annu Rev Immunol 17: 19-49, 1999.

39. Iizuka K, Chaplin DD, Wang Y, et al: Requirement for membrane lymphotoxin in natural killer cell development. Proc Natl Acad Sci USA 96: 6336-6340, 1999. 